\title{
ACCELERATION OF ULTRA HIGH ENERGY COSMIC RAYS
}

\author{
R. D. Blandford \\ 130-33 Caltech \\ Pasadena CA 91125 USA
}

August 24, 2018

\begin{abstract}
Some general features of cosmic ray acceleration are summarized along with some inferences that can be drawn concerning the origin of the UHE component. The UHE luminosity density is found to be similar to that derived for $\mathrm{GeV}$ cosmic rays and its slope suggests a distinct origin. Reports of clustering on small angular scale, if confirmed, would rule out most proposed source models. More generally, it is argued that the highest energy particles can only be accelerated in sites that can induce an EMF $\mathcal{E} \gtrsim 3 \times 10^{20} \mathrm{~V}$ and an associated power $L_{\text {min }} \gtrsim \mathcal{E}^{2} / Z \sim 10^{39} \mathrm{~W}$, where $Z$ is the characteristic, electrical impedance, typically $\lesssim 100 \Omega$. Shock acceleration, unipolar induction and magnetic flares are the three most potent, observed, acceleration mechanisms and radio jet termination shocks, $\gamma$-ray blast waves, dormant black holes in galactic nuclei and magnetars are the least implausible, "conventional" manifestations of these mechanisms that have been invoked to explain the UHE cosmic rays. Each of these models presents problems and deciding between these and "exotic" origins for UHE cosmic rays, including those involving new particles or defects will require improved statistical information on the energies, arrival times and directions, as should be provided by the Auger project.
\end{abstract}




\section{INTRODUCTION}

I have been asked to summarize "conventional" schemes for the acceleration of UHE cosmic rays, though any physical process capable of endowing a subatomic particle with the kinetic energy of a well-hit baseball/cricketball can hardly be considered conventional. This means that I shall leave others to review mechanisms that attribute the origin of these particles to topological defects, strings, monopole decay, supersymmetric hadrons, cosmic necklaces, cryptons and so on. Indeed, I suspect that the "hidden agenda" is for me to fail at my appointed task, and, like my colleagues on the MACHO experiment, to make the world safe for elementary particle theorists. I shall not disappoint.

Many of the issues that I will cover have been recognised for some time and have been well-discussed in several excellent reviews including Hillas (1984) and Cronin (1996) and the many relevant contributions to the recent conference on this subject (Krizmanic, Ormes \& Streitmatter 1998), including, especially, the lively summary by the late David Schramm. The conference proceedings edited by Chupp \& Benz (1994) is also relevant.

\section{THE COSMIC RAY SPECTRUM}

In order to give this topic some context, consider the complete cosmic ray spectrum (eg Berezinski et al 1990). This extends over nearly twelve decades of energy from the proton rest mass, $\sim 1 \mathrm{GeV}$, where their energy density is that of the microwave background, to at least $300 \mathrm{EeV}$ ( $\equiv 50 \mathrm{~J} \equiv 3 \times$ $\left.10^{-8} \mathrm{~m}_{\mathrm{Pl}}\right)$. We can consider the cosmic ray spectral energy density inferred at the solar system, $U(E)=(4 \pi / c)(d I / d \ln E) \sim 5 \times 10^{-14}(E / 10 \mathrm{GeV})^{-0.7} \mathrm{~J}$ $\mathrm{m}^{-3}$ (correcting for solar modulation) extending from $\sim 10 \mathrm{GeV}$ to the "knee" at $\sim 100 \mathrm{TeV}-10 \mathrm{PeV}$. (10 GeV cosmic rays are about $10 \mathrm{~m}$ apart and have an energy density comparable with that of the microwave background. The spectrum steepens above the knee: $U(E) \sim 4 \times 10^{-18}(E / 10 \mathrm{PeV})^{-1.1} \mathrm{~J} \mathrm{~m}^{-3}$. It then dips and flattens around the "ankle" $(\sim 1-10 \mathrm{EeV})$. UHE cosmic rays - the toenail clippings of the universe - are observed up to $300 \mathrm{EeV}$ and, with a little imagination, $U(E) \sim 1.5 \times 10^{-21}(E / 10 \mathrm{EeV})^{-0.5} \mathrm{~J} \mathrm{~m}^{-3}$, comparable with the estimated, integrated background from $\gamma$-ray bursts. (Despite the large uncertainty, and the fact that the number density has fallen by $\sim 10$ 
orders of magnitude, we do measure the EeV spectrum better than the $\mathrm{MeV}$ spectrum, of which we are, quite decently, ignorant.)

The $\sim 1 \mathrm{GeV}-100 \mathrm{TeV}$ cosmic rays are of Galactic origin. The ratio of Li, Be, B secondaries to $\mathrm{C}, \mathrm{N}, \mathrm{O}$ primaries measures their range to be $\lambda(E) \sim$ $100(E / 10 \mathrm{GeV})^{-0.6} \mathrm{~kg} \mathrm{~m}^{-2}$ (eg Axford 1994). The cosmic ray luminosity of the Galaxy is then estimated as $\sim M_{d} U(E) c / \lambda(E) \sim 2 \times 10^{33}(E / 1 \mathrm{GeV})^{-0.1} \mathrm{~W}$ where $M_{d}$ is the gas mass of the disk. Scaling from the local galaxy luminosity density (per $\ln E$ and assuming $h=0.6$ ), we derive an average, cosmological, luminosity density (per $\ln E),<\mathcal{L}>(E) \sim 4 \times 10^{-37}(E / 10 \mathrm{Gev})^{-0.1} \mathrm{~W} \mathrm{~m}^{-3}$, for $10 \mathrm{GeV}<E<100 \mathrm{TeV}$. (For comparison, the stellar luminosity density is $\sim 10^{-33} \mathrm{~W} \mathrm{~m}^{-3}$.)

The UHE particles are almost surely extragalactic. As with $\gamma$-ray bursts, there is no good evidence for disk, halo, cluster or supercluster anisotropy (despite some tantalising hints in the past), (Takeda et al 1999). Furthermore, magnetic confinement by the Galaxy is impossible - the Larmor radius $r_{L}(E)$ of a $300 \mathrm{EeV}$ cosmic ray in a $\mu \mathrm{G}$ field is $\sim 300 \mathrm{kpc}$. If we assume that UHE cosmic rays are protons, (and assuming that they are not, only makes matters worse), then they have a short lifetime to photo-pion production on the microwave background, (Greisen 1966, Zatsepin \& Kuzmin 1966). The characteristic lifetime of a $\sim 60-300 \mathrm{EeV}$ cosmic ray is, very roughly, $T(E) \sim 0.1(E / 300 \mathrm{EeV})^{-2}$ Gyr. This implies that the luminosity density increases with energy $\left\langle\mathcal{L}>(E) \sim U(E) / T(E) \sim 10^{-37}(E / 300 \mathrm{EeV})^{1.5} \mathrm{~W}\right.$ $\mathrm{m}^{-3}$. At the highest measured energy, the estimated cosmological luminosity density is not significantly different from that of $10 \mathrm{GeV}$ cosmic rays. The change in slope in the source spectrum, above $\sim 60 \mathrm{EeV}$, is a strong indication that these UHE cosmic rays comprise a quite distinct component from their lower energy counterparts.

In order to investigate this further, it is necessary to take account of the fluctuations in energy loss. Taking the 17 events reported by the AGASA collaboration above $60 \mathrm{EeV}$, it is possible to derive a maximum likelihood estimate of the unnormalized energy density, uncorrected for biases in detection efficiency. I find that if $U(E) \propto E^{\alpha} ; E>E_{\min }=60 \mathrm{EeV}$, then $\alpha=-1.2 \pm 0.5$. I then calculate the probability that a particle of energy $E_{0}$ has energy $>E$ after time $t, P\left(E, t ; E_{0}\right)$, following Aharonian \& Cronin (1994) and Bahcall \& Waxman (1999). If we assume a power law for the luminosity density $\mathcal{L}(E) \propto E^{\beta} ; E>E_{\text {min }}=60 \mathrm{EeV}$, then the logarithm of 
the likelihood for obtaining the observed events is

$$
\propto \sum_{i} \ln \left[(1-\beta) E_{\min }^{(1-\beta)} \int_{E_{i}} d E_{0} E_{0}^{\beta-2} \int d t \frac{\partial P}{\partial \ln E}\right]
$$

Maximizing this function with respect to variation of $\beta$, gives the estimate $\beta=0.3 \pm 0.2$. A more sophisticated computation that takes into account the detection probabilities of the different events should be performed, but it is unlikely to change the conclusion that the spectral luminosity density actually increases in the 60-300 EeV energy range and may even be consistent with a single, "top down" source with energy well above $300 \mathrm{EeV}$.

There have been reports that UHE cosmic rays are significantly clustered on the sky. Specifically, in a sample of 47 events observed with AGASA, there are three pairs and one triple above $40 \mathrm{EeV}$ with separations $\lesssim 2.5^{\circ}$, comparable with the positional errors (Takeda et al 1999). (There are two more coincidences with events drawn from other samples.) There is no clear pattern for the associated particles to be ordered in energy and, in particular, one double has a 106EeV particle arriving over 3 yr. after a $44 \mathrm{EeV}$ particle.

If these associations are real, then there are three important implications. Firstly, as particles are likely to be deflected by intergalactic magnetic field through an angle $\delta \theta \sim\left(D \ell_{B}\right)^{1 / 2} / r_{L}(E)$ then they will be delayed by $\sim D^{2} \ell_{B} / r_{L}(E)^{2} c \propto E^{-2}$, where $\ell_{B}$ is the field correlation length and $D \sim c T(150 \mathrm{EeV}) \sim 30 \mathrm{Mpc}$ is the supposed source distance ( cf MiraldaEscudé \& Waxman 1996). Even Aesop would be challenged to explain how a $\sim 40 \mathrm{EeV}$ cosmic ray precedes a $\sim 100 \mathrm{EeV}$ cosmic ray if they started at the same time and we must conclude that the source persists for several years, at least. This would rule out all particle/defect and $\gamma$-ray burst models. Secondly, the small deflection angles at low energy limit the intergalactic field strength to $B \lesssim 20\left(\ell_{B} / 1 \mathrm{Mpc}\right)^{-1 / 2}$ fT, far smaller than generally supposed, though probably not excludable by direct observation. Thirdly, the presence of three $\sim 40 \mathrm{EeV}$ cosmic rays associated with high energy cosmic rays of much shorter range, implies that the background of low energy cosmic rays not associated with high energy events must be larger than the incidence of clustered events by roughly the ratio of their typical lifetimes $\sim 30$, which more than accounts for the remainder of the low energy sample. This, in turn, implies that the high energy cosmic rays must come from a very few sources which are, consequently, quite energetic: $E \sim 10^{44}(\tau / 3 \mathrm{yr}) \mathrm{J}$, where $\tau$ 
is their lifetime. (If the low energy cosmic rays are scattered through $\sim 2.5^{\circ}$, then $\tau \gtrsim 10^{5}$ yr and $E \sim 3 \times 10^{48} \mathrm{~J}$.)

However, this clustering hypothesis, which is necessarily a posteriori when expressed in detail, is only supported with modest confidence. (A simple, Monte Carlo simulation distributing 47 points at random on half the sky and looking for similar patterns is quite instructive.) Particle/defect/burst explanations of UHE cosmic rays need not yet be rejected on these grounds.

\section{COSMIC RAY ACCELERATION}

The standard model of bulk cosmic ray production is first order Fermi acceleration at strong, super-Alfvénic, shocks associated with supernova remnants and, possibly, winds from hot stars (eg Blandford \& Eichler 1987). A typical relativistic proton will cross a shock, travelling with speed $u, O(c / u)$ times, gaining energy $\Delta E / E=O(u / c)$ each traversal through scattering by hydromagnetic waves moving slowly with respect to the converging fluid flows on either side of the front. The net mean relative energy gain is $O(1)$, but the process is statistical and a kinetic calculation shows that the transmitted

spectrum will be a power law in momentum, $f(p) \propto p^{-3 r /(r-1)}$, where $r,(=4$ for a strong shock), is the compression ratio. This mechanism can account, broadly, for the power (eg Malkov 1999), the slope (eg Axford 1994) and the composition (eg Ellison et al (1997)) of GeV cosmic rays. Shock acceleration is also, arguably, observed directly in SN1006 (Koyama et al 1995), as well as in the solar system ( $e g$ Erdös \& Balogh 1994).

The maximum energy to which a particle can be accelerated at a shock front is dictated by the scattering mean free path, $\ell(E) \sim(B / \delta B)_{r_{L}}^{2} r_{L}(E)$, where $\delta B$ is the amplitude of resonant hydromagnetic waves with wavelength matched to the particle Larmor radius. The diffusion scale-length of cosmic rays ahead of the shock is $\sim \ell c / u$ and, assuming that this is limited by the size of the shock $\sim R$ we arrive at the unsurprising result that the maximum energy achievable in shock acceleration, assuming $\delta B<B$ and the presence of a large scale magnetic field, is $E_{\max }=e \mathcal{E} \sim e u B R \sim e d \Phi / d t$, the product of the charge and the motional potential difference across the whole shock. Equivalently, we conclude that in order to accelerate a proton by this mechanism to an energy $\mathcal{E} e$, the rate of dissipation of energy exceeds $L_{\text {min }} \sim \mathcal{E}^{2} / Z$, where $Z=\mu_{0} u=\mu_{0} E / B$ is the effective impedance of the 
accelerator in SI units.

Imposing this condition for a supernova remnant in the interstellar medium leads to an estimate $E_{\text {maxsnr }} \sim 30 \mathrm{TeV}$ ( eg Axford 1994), close to the knee. An additional source is needed between the knee and the ankle, where the source is generally supposed to be metagalactic. Larger shocks, especially those at Galactic wind termination shocks (eg Jokipii \& Morfill 1987) and associated with gas flows around groups and clusters of galaxies have been invoked (eg Norman et al 1995). These shocks are likely to be relatively weak and therefore to transmit steeper spectra, as observed. The major uncertainty is the strength of the magnetic field. If $B \sim 30 \mathrm{pT}$ at a galactic shock and $\sim 10$ pT at a cluster shock, then $E_{\text {max }} \sim 10,100 \mathrm{PeV}$ respectively. Neither site is likely to accelerate the highest energy particles.

An alternative accelerator is the unipolar inductor (eg Goldreich \& Julian 1967). The archetypical example is a pulsar - a spinning, magnetised, neutron star. The surface field will be quite complex but a certain quantity of magnetic flux $\Phi$ can be regarded as "open" and tracable to large distances from the star, (well beyond the light cylinder). As the star is an excellent conductor, an EMF will be electromagnetically induced across these open field lines $\mathcal{E} \sim \Omega \Phi$, where $\Phi$ is the total, open magnetic flux. This EMF will cause currents to flow along the field and as the inertia of the plasma is likely to be insignificant the only appreciable impedance in the circuit is related to the electromagnetic impedance of free space $Z \sim 0.3 \mu_{0} c \sim 100 \Omega$. The maximum energy to which a particle can be accelerated is $E_{\max } \sim e \mathcal{E}$ and the total rate at which energy is extracted from the spin of the pulsar is $L_{\min } \sim \mathcal{E}^{2} / Z$. Taking the Crab pulsar as an example, $E_{\max } \sim 30 \mathrm{PeV}$ for protons and $L_{\min } \sim 10^{31} \mathrm{~W}$. As the stellar surface may well comprise iron, even the Crab pulsar has the capacity to accelerate up to $\mathrm{EeV}$ cosmic rays. However, it is not obvious that all of this potential difference will actually be made available for particle acceleration. In particular, this is unlikely to happen in the pulsar magnetosphere as a large electric field parallel to the magnetic field will be shorted out by electron-positron pairs, which are very easy to produce, and radiative drag is likely to be severe. A more reasonable site is the electromagnetic pulsar wind and the surrounding nebula where particles can gain energy as they undergo gradient drift between the pole and the equator (Bell 1992). Pulsars may well contribute to the spectrum of intermediate energy cosmic rays.

A third, protoypical accelerator is a flare, for example one occuring on the 
solar surface or the Earth's magnetotail. Here magnetic instability leads to a catastrophic rearrangement, which must be accompanied by a large inductive EMF. Unless the instabilities are explosive, the effective impedance is again $\sim \mu_{0} u$, where $u$ is a characteristic speed. Non-relativistic flares generally convert most of the dissipated magnetic energy into heat and are notoriously inefficient in accelerating high energy particles.

Other acceleration mechanisms have been proposed and may contribute to the acceleration of the bulk of Galactic cosmic rays and relativistic electrons in non-thermal sources. These include a variety of second order processes and steady, magnetic reconnection. Many of them can be observed to operate within the solar system. However, they are thought to be too slow to be relevant to the acceleration of the highest energy cosmic rays.

\section{ZEVATRONS}

Having argued that the three most potent, observed acclerators are shocks, unipolar inductors and flares, let us see how they can be modified to account for $\sim \mathrm{ZeV}$ cosmic rays. Firstly, note that, as $u \sim c$, mildly relativistic shocks minimise the power that has to be invoked to attain high energy. Specifically, we need a power $>10^{39} \mathrm{~W}$ to account for $300 \mathrm{EeV}$ cosmic rays and this exceeds the bolometric luminosity of a powerful quasar. One of the few sites where such a large potential difference can be achieved is the termination shock of a powerful radio jet like that associated with Cygnus A (eg Cavallo 1978). Stretching the numbers a little, we combine a field strength $\sim 10 \mathrm{nT}$, with a speed $\sim c$ and a transverse scale $\sim 3 \mathrm{kpc}$ which gives $E_{\max } \sim 300 \mathrm{EeV}$. The problem with this model is that observed UHE cosmic rays are not positionally identified with the few known radio sources within $D(E) \sim 30 \mathrm{Mpc}$ that might be powerful enough to account for them (cf Farrar \& Biermann 1998).

A more elaborate shock accelerator is the $\gamma$-ray burst blast wave (eg Waxman 1995). Here, the shocks (assumed to be spherical) are ultrarelativistic with Lorentz factor $\Gamma$. The maximum energy, measured in the frame of the explosion, to which a proton can be accelerated in a dynamical timescale from an ultrarelativistic shock of radius $R$ is $E_{\max } \sim e B^{\prime} R c$, where $B^{\prime}$ is the comoving field strength. The explosion power, adopting the most elementary of assumptions, is then $L_{\min } \sim 4 \pi \Gamma^{2}\left(E_{\max } / e\right)^{2} / \mu_{0} c$. Observed bursts 
have typical explosion powers estimated to be $L_{\exp } \sim 10^{45} \mathrm{~W}$, which can be consistent with $300 \mathrm{EeV}$ proton acceleration as long as $\Gamma<300$, which is just compatible with existing models. A serious physical constraint is the avoidance of radiative loss in this environment. An observational concern with this model is the improbability of having enough active bursts close to supply the highest energy particles roughly isotropically (cf Waxman \& Miralda-Escudé 1996).

The most relevant variant on unipolar induction is magnetic energy extraction from spinning, black holes, where the magnetic field is supported by external current, and the horizon is an imperfect conductor with resistance $\sim 100 \Omega$ (eg Thorne it et al 1986). This impedance is matched to the electromagnetic load so that roughly half of the available spin energy ends up in the irreducible mass of the hole, the remainder being made available for particle acceleration. The total electromagnetic power needed to account for $\sim 300 \mathrm{EeV}$ acceleration is, once more, $\sim 10^{39} \mathrm{~W}$. A rapidly spinning, $\sim 10^{9} \mathrm{M}_{\odot}$ hole endowed with a field strength $\gtrsim 1 \mathrm{~T}$ or a $\sim 10^{5} \mathrm{M}_{\odot}$ hole threaded by a $\gtrsim 10^{4} \mathrm{~T}$ field suffices to accelerate $300 \mathrm{EeV}$ particles. The major concern with this model is that the radiation background must be extremely low in order that catastrophic loss due to pion and pair production be avoided. Specifically, it is necessary that the microwave luminosity in an acceleration zone, of size $R$, be $\lesssim 10^{34}\left(R / 10^{14} \mathrm{~m}\right) \mathrm{W}$, far smaller than the unobserved electromagnetic power.

The best generalization of flare acceleration involves "magnetars" which are young, spinning neutron stars endowed with a $\sim 10-100 \mathrm{GT}$ surface magnetic field as first postulated by Thompson \& Duncan, (1996). Now, the observation of $5-7$ s period pulsations from three "soft gamma repeaters" effectively confirms their identification as old magnetars that have been decelerated by electromagnetic torque and which are now powered by magnetic energy which is released in a series of giant flares, Kouveliotou et al 1998. The inductive EMFs associated with an electromagnetic flare from a magnetar can be as high as $\mathcal{E} \sim 3 \times 10^{19} \mathrm{~V}$, making them candidate UHE accelerators because the surface composition is likely to be Fe. However, the available reservoir of magnetic energy is only $\sim 10^{40} \mathrm{~J}$ and the magnetar birthrate is no more than $\sim 10^{-3} \mathrm{yr}^{-1}$ in the Galaxy. This rules them out as an extragalactic source. Only if UHE cosmic rays have a Galactic origin, (and the large scale anisotropy observations suggest quite strongly that do not), can there be enough power in magnetars to account for the UHE energy 
density.

\section{DISCUSSION}

I have argued, tentatively, that UHE cosmic rays are created in a new population of extragalactic sources with an average luminosity density that approaches that of Galactic cosmic rays. I have also described problems with each of the candidate "conventional" mechanisms for accelerating protons to these high energies. Quite different, general inferences have been drawn here, from the same data, by Waxman, and elsewhere by others. All of this underscores the need for better statistics which should be met by the Auger project. Perhaps the most pressing need is to understand if particles of very different energy have a common origin. If true, this must rule out essentially all primordial particle/topological defect, neutron star, $\gamma$-ray burst explanations, leaving only massive black holes and radio source models among the possibilities discussed above. In this case, it will be possible to seek identifications, especially at the highest energies, where the positions will be most accurate and the delays due to magnetic scattering the smallest. If, alternatively, clustering and its implications are not substantiated, then the next best clues will probably come from composition studies and detailing the large scale distribution on the sky.

The most exciting outcome of all of this is that we are dealing with a new particle or defect with energy well out of the range of terrestrial accelerators. (For example, if there is a particle of energy $E_{X}$ which decays with half life $\tau_{X}$ into $N$ protons, then the cosmological energy density of these particles must be $\Omega_{X} \sim 3 \times 10^{-8} N^{-1}\left(E_{X} / 1 \mathrm{YeV}\right)^{-1}\left(H_{0} \tau_{X}\right)^{-1}$.) Whatever happens, in a subject where the dullest and most conventional theories involve massive, spinning, black holes, ultrarelativistic blast waves and 100 GT fields threading nuclear matter, the future is guaranteed to be interesting.

\section{Acknowledgements}

I am indebted to John Bahcall, Jim Cronin, Michael Hillas, Martin Rees, Alan Watson and Eli Waxman for stimulating discussions and the editors for their forbearance. I also gratefuly acknowledge the hospitality of the Insitute 
for Advanced Study (through the Sloan Foundation) and the Institute of Astronomy (through the Beverly and Raymond Sackler Foundation) as well as NASA grant 5-2837.

\section{References}

[1] Norman, C. A., Melrose, D. B. \& Achterberg, A. ApJ 199545460

[2] Aharonian, F. \& Cronin, J. 1994 PRD 501892

[3] Axford, W. I. 1994 ApJS 90937

[4] Bahcall, J. N. \& Waxman, E. 1999 (preprint)

[5] Bell, A. R. 1992 MNRAS 257493

[6] Berezinski, V. S. et al 1990 Astrophysics of Cosmic Rays Amsterdam:North Holland

[7] Blandford, R. D. \& Eichler, D. 1987 Phys. Rep. 1541

[8] Chupp, E. L. \& Benz, A. O. (ed.) 1994 ApJS 90511

[9] Farrar, G. R. \& Biermann, P. L. 1998 PRL 813579

[10] Cavallo, G. 1978 A \& A 65415

[11] Cronin, J. Unsolved Problems in Astrophysics ed. J. Bahcall \& J. Ostriker Princeton:Princeton University Press p 325

[12] Ellison, D., Drury, L. O’C. \& Meyer, J.-P. 1997 ApJ 487197

[13] Erdös, G. \& Balogh, A. 1994 ApJS 90553

[14] Goldreich, P. \& Julian, W. H. 1967 ApJ 157869

[15] Greisen, K. 1996 PRL 16748

[16] Hillas, M. 1984 ARAA 22245

[17] Jokipii, J. R. \& Morfill, G. 1987 ApJ 312170 
[18] Kouveliotou, C. et al 1998 Nature 393235

[19] Koyama, K. et al 1995 Nature 378255

[20] Krizmanic, J. F., Ormes, J. F. \& Streitmatter, R. E. ed. 1998 New York:AIP

[21] Malkov, M. A. 1999 ApJ 511 L53

[22] Miralda-Escudé, J. \& Waxman, E. 1996 ApJ 462 L59

[23] Takeda, M. et al 1999 astro-ph/9902239

[24] Thompson, C. A. \& Duncan, R. C. 1995 MNRAS 275255

[25] Thorne, K. S., Price, R. M. \& MacDonals, D. 1986 Black Holes: The Membrane Paradigm New Haven: Yale University Press

[26] Waxman, E. 1995 PRL 75386

[27] Waxman, E. \& Miralda-Escudé, J. 1996 ApJ 472489

[28] Zatsepin, G. T. \& Kuz'min, V. A. 1966 JETP 4 L78 